\title{
Saber vivir allí como principio de vida Inga
}

\author{
Knowing How \\ to Live There as \\ a Principle of the \\ Inga Life \\ Saber viver lá \\ como princípio de \\ vida Inga
}

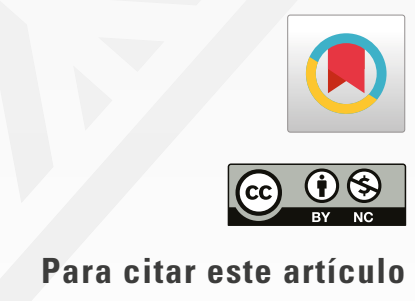

Bravo Osorio, M. (2021). Saber vivir allí como principio de vida Inga. Folios, 53. https://doi.org/10.17227/folios.53-10183

Candidata a doctora en Etnobiología y estudios bioculturales de la Universidad del Cauca y magíster en Estudios Sociales. Docente investigadora Grupo de investigación Enseñanza de la biología y diversidad cultural. Profesora ocasional de tiempo completo Universidad Pedagógica Nacional.

Correo electrónico: Imbravoo@pedagogica.edu.co. 


\title{
Resumen
}

Este artículo de investigación presenta la caracterización del principio “saber vivir allí", desde la mirada del pueblo indígena Inga de la Baja Bota Caucana (Colombia) y su relación con las posibilidades de cuidado del territorio, en diálogo con perspectivas filosóficas andinas y con el paradigma del cuidado de la vida. La propuesta metodológica en este ejercicio investigativo es construida a partir de la experiencia de vida con los Inga, entendida como posibilidad de desenvolver conocimientos que aportan a las lecturas de realidad desde los estudios sociales. Planteando una ruta metodológica con enfoque cualitativo y crítico, que comprende las experiencias de vida, los diálogos, los registros audiovisuales y la sistematización y análisis de la información. Como principal hallazgo se presenta el "saber vivir allí" como un principio de vida Inga, que no hace referencia únicamente a formas humanas de sobrevivencia, sino fundamentalmente a entender que hacemos parte de algo más grande, tan complejo en su existencia como nosotros mismos: el territorio. Lleno de otras formas de saber vivir, con las cuales debemos relacionarnos y buscar la complementariedad, reciprocidad y armonía. Configurándose como alternativa de vida y de resistencia frente a las formas capitalistas que desvirtúan principios de armonización de la vida, de solidaridad e igualdad.

\section{Palabras clave}

Pueblo Inga; saber vivir allí; territorio; conocimiento

\begin{abstract}
This research article presents the characterization of the principle "knowing how to live there", from the perspective of the Inga indigenous people of the Baja Bota Caucana (Colombia) and its relationship with the possibilities of caring for the territory, in dialogue with Andean philosophical perspectives and with the paradigm of the care of life. The methodological proposal in this research initiative is based on the experience of living with the Inga, understood as the possibility of developing knowledge that contributes to the readings of reality from social studies. The methodology proposed follows a qualitative and critical approach, which includes life experiences, dialogues, audiovisual records and the systematization and analysis of information. The main finding is the "knowing how to live there" as a principle of Inga life, which does not refer only to human survival forms, but fundamentally to understand that we are part of something bigger, as complex in its existence as ourselves: the territory. It is full of other ways of knowing how to live, with which we must relate and seek complementarity, reciprocity, and harmony. It settles as an alternative of life and resistance to capitalist forms that undermine principles of harmonization of life, solidarity, and equality.
\end{abstract}

$$
\begin{array}{r}
\text { Keywords } \\
\text { Inga people; knowing how to live there; territory; knowledge }
\end{array}
$$

\section{Resumo}

Este trabalho de pesquisa apresenta a caracterização do princípio "sabe viver lá", a partir da perspectiva dos povos indígenas da Baja Bota Caucana Inga (Colômbia) e sua relação com as possibilidades do cuidado do território, em diálogo com perspectivas filosóficas andinas e com paradigma do cuidado da vida. A proposta metodológica neste exercício de pesquisa baseia-se na experiência de conviver com o Inga, entendida como a possibilidade de desenvolver conhecimentos que contribuam para as leituras da realidade a partir dos estudos sociais. Propondo um percurso metodológico com abordagem qualitativa e crítica, que inclui experiências de vida, diálogos, registros audiovisuais e sistematização e análise de informações. A principal constatação é o "sabe viver lá" como princípio da vida Inga, que não se refere apenas às formas de sobrevivência humana, mais fundamentalmente entender que somos parte de algo maior, tão complexo em sua existência quanto a nós mesmos: o território. Ele está cheio de outras formas de saber viver, com as quais devemos nos relacionar e buscar complementaridade, reciprocidade e harmonia. Estabelecendo-se como uma alternativa de vida e resistência às formas capitalistas que minam os princípios de harmonização da vida, solidariedade e igualdade.

\section{Palavras chave}

povo Inga; sabe viver lá; território; conhecimento 


\section{Introducción}

Este artículo desarrolla elementos que aportan a la comprensión del "saber vivir alli"', expresión recurrente en la cotidianidad de la comunidad indígena Inga, ${ }^{2}$ ubicada en la Baja Bota Caucana, al sur de Colombia, como un modo particular de estar en el territorio, de recrear la vida y, por esa vía, cuidarla.

A partir de lo anterior, se identifica, por un lado, la intención de explorar desde ellos lo que implica este principio, como una noción que expresa su relación con los seres y existencias que habitan nukanchipa alpa mama (nuestro territorio); por el otro, relacionarlo con unas condiciones particulares del contexto que habitan.

Para el caso de los Inga de la Baja Bota Caucana (вBC), habitan un territorio caracterizado por el hecho de estar ubicados en el piedemonte amazónico, el cual es considerado:

un lugar de encuentro: mezcla entre la margen y el centro; un espacio tan urbano, desarrollado y 'civilizado' como el centro, pero también, tan rural, atrasado y 'salvaje' como la margen... el piedemonte, como todo lugar de frontera, es un espacio de mezcla, es un crisol donde se funden diferentes valores territoriales y culturales. (Ciro y Ciro, 2008, p. 107)

Precisamente esa diversidad de valores territoriales y culturales es uno de los elementos que genera tensiones entre la variedad de actores sociales que hacen presencia en la zona de estudio: campesinos, colonos, instituciones estatales y no estatales, actores armados legales e ilegales, comunidades

1 Reflexiones que hacen parte de los resultados de la investigación titulada: Ugpachisunchi i katichisunchi kilkaikunata (llevando y trayendo la palabra): territorio, "saber vivir ahí" y pensamiento Inga, realizada para optar al título de magíster en Estudios Sociales.

2 El pueblo Inga es una comunidad indígena de Colombia, ubicada principalmente en los departamentos del sur de nuestro país (Nariño, Putumayo, Cauca y Caquetá). También hace presencia en Villavicencio, Bogotá, Medellín, Cali, Yopal, Arauca, Puerto Carreño, Valledupar, Santa Marta, Cartagena, Barranquilla, Bucaramanga, Riohacha, Cúcuta y Buenaventura (Pueblo Inga, 2013) y "compartimos pensamiento, idioma, tradiciones y una historia de origen común que nos hermana con otros pueblos del Ecuador, Perú, Bolivia, norte de Chile y Argentina..." (Pueblo Inga - Ministerio de Educación Nacional, 2012).

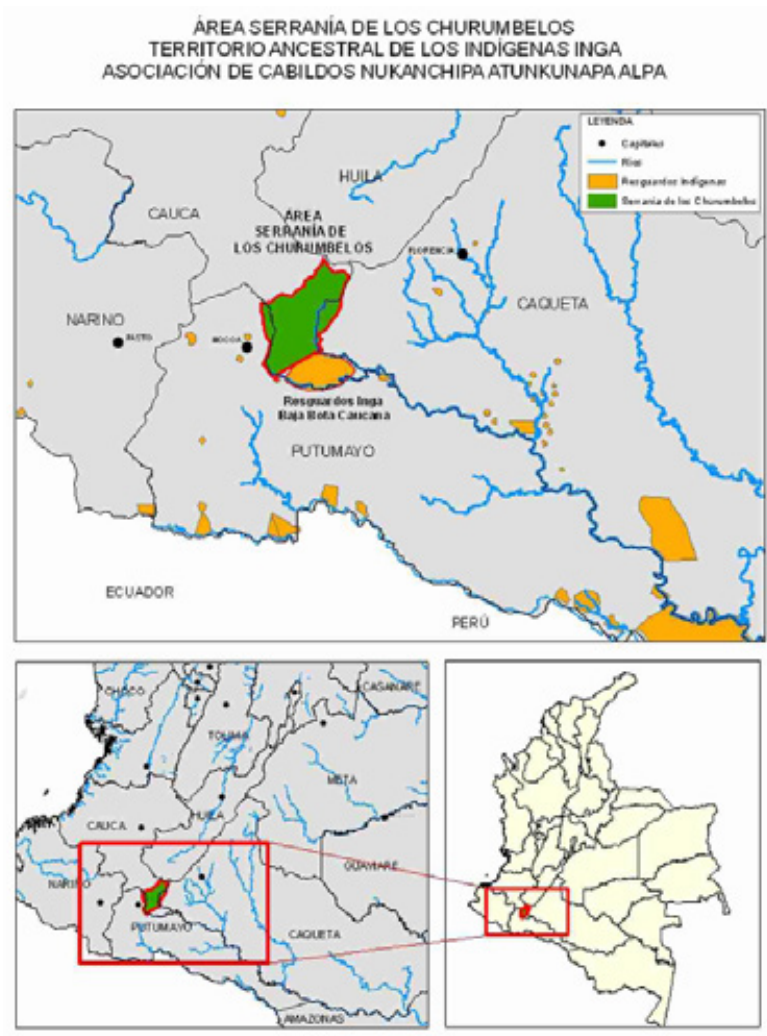

llustración 1. Ubicación geográfica de la Baja Bota Caucana.

Fuente: Pueblo Inga, 2012 Plan de salvaguarda del Pueblo Inga de Colombia: Nukanchipa Kaugsaikunata luiaikunata Mana Uañungapa Sakisunchi. Mocoa.

afrodescendientes e indígenas de diferentes etnias. Tensiones que según el plan de salvaguarda del pueblo Inga (2013) se expresan en:

- [Presencia de] compañías petroleras y mineras que al explotar los recursos naturales, no solo causan un impacto negativo en nuestra naturaleza, fauna y flora, sino que también amenazan la estabilidad de nuestra madre tierra y la de la comunidad.

- Los cultivos ilícitos como la coca.

- Las fumigaciones con glifosato y otros químicos.

- La presencia de grupos armados legales [ejército nacional de Colombia, frentes contra-guerrilla] e ilegales [bloque sur de la guerrilla de las Fuerzas Armadas Revolucionarias de Colombia (FARC), frente 32, además de 
estar en el área de influencia de grupos paramilitares, como los Macheteros y rastrojos (Pardo, 2007, p. 159)] en nuestro territorio. ${ }^{3}$

- La presencia de instituciones que vienen con diferentes tipos de intereses, particulares en su haber.

- Pérdida de pertenencia y de la relación Ingamadre naturaleza.

- Actualmente nuestro territorio se va dividiendo en hectáreas, metros cuadrados, cada vez el Estado nos va quitando el espacio en que anteriormente nuestros mayores se desplazaban con tranquilidad, era un gran territorio que no tenía límite. Ahora tenemos que comprar tierra para extender nuestro territorio. Así, la pérdida de territorio se convierte, no solo en una problemática, sino en una amenaza para nuestra pervivencia como pueblo y etnia. Sumado a esto, nos enfrentamos a: desplazamiento forzoso, colonización, invasión de territorio ancestral, falta de saneamiento básico, presencia de grupos armados legales e ilegales (p. 81).

En este panorama el principio de vida Inga: "saber vivir allí" se toma como elemento que aporta a las lecturas de realidad de vida por las que atraviesan como comunidad indígena en relación con las dinámicas económicas y políticas de Colombia. La apuesta de este artículo es visibilizar las prácticas de vida que este pueblo indígena recrea a partir de su cotidianidad como aporte para el cuidado de sus vidas, y la diversidad biológica y cultural de sus territorios.

\section{Metodología}

Esta investigación es de corte cualitativo con un enfoque de los estudios sociales. Uno de los aspectos a destacar es el interés por construir las lecturas de realidad a partir del diálogo con los contextos, realidades y actores de la investigación. Específicamente retomando prácticas culturales del

3 Para el año 2015, momento en el que se termina esta investigación, aún se contaba con presencia de los actores armados de la guerrilla de las Farc. Con la firma del acuerdo entre el gobierno colombiano y esta guerrilla en el año 2016, estos actores despejan la zona. pueblo Inga, como caminar, conversar alrededor de la tulpa (fogón) y las ceremonias de medicina ancestral con el bejuco de Ambiwaska, como posibilidades de construcción colectiva de conocimientos. Se han retomado herramientas para la recolección de la información como el diario de campo, las entrevistas y conversatorios con la comunidad y para la sistematización y análisis de la información, la categorización relacional y, a partir de esta, la construcción de los textos presentados.

\section{Referente conceptual}

\section{Territorio}

Los debates conceptuales alrededor de esta categoría de estudio dan cuenta de la polisemia de sus significados. Específicamente desde la perspectiva de esta investigación se propone asumirlo como una categoría que permite pensarlo como un espacio de vida, configurado a partir de las relaciones sociales que se dan en un espacio particular; lo cual implica las relaciones cultura-natura, desde las cosmovisiones y cosmogonías de los diferentes grupos sociales que lo habitan. Esto conlleva a entender el territorio, más allá del espacio geográfico, contemplando para su estudio las tensiones que surgen al estar habitado por distintos actores sociales, cada cual con sus particulares sentires y configuración de mundo.

En este sentido, se evidencia cercanía en los debates conceptuales que proponen autores como Bozano (2000), donde el territorio constituye una construcción social e histórica, un híbrido entre naturaleza y sociedad, enfocando su atención en las relaciones que surgen a partir de la vida en un lugar particular. Para el caso de los territorios indígenas, en particular, es importante posicionar miradas territoriales que los comprenden como categoría emergente de las interacciones entre los subsistemas sociales y físico-naturales, que necesariamente dialogan con las condiciones políticas, culturales y económicas particulares y que, por tanto, implican abordajes desde miradas complejas inter y transdisciplinares.

En este sentido, conceptos como territorio, ambiente y región "son expresiones de la especialización del poder y de las relaciones de cooperación 
o de conflicto que de ellas se derivan" (Delgado y Montañez, 2007, p. 56). Es en esa dirección que pensar en el territorio, como concepto estructurante de esta investigación, implica ubicarlo en diálogo no solo con los debates académicos, sino también con nociones y sentidos que se han venido apropiando y construyendo desde las comunidades con las cuales se va a trabajar.

Vasco (2000), plantea entender el territorio sin hacer exclusivo énfasis en las relaciones "directamente económicas -de propiedad y producción-", ya que estas solo son una más dentro del complejo relacional que constituye el territorio, por lo cual es importante también contemplar la diversidad de relaciones mediante las cuales se apropian, utilizan y piensan dicho espacio, socializándolo.

Dentro de este marco general de análisis, se propone para este trabajo entender el territorio desde una perspectiva sistémica donde sea considerado como una propiedad emergente, que surge de la "apropiación que se da del espacio geográfico" a través del pensamiento y la palabra, sin desconocer su dimensión histórica y espacial. Es decir, como algo que no se limita a la suma de sus partes, pero tampoco existiría sin estas.

\section{Resultados y discusión}

\section{"Saber vivir alli" como práctica de vida Inga}

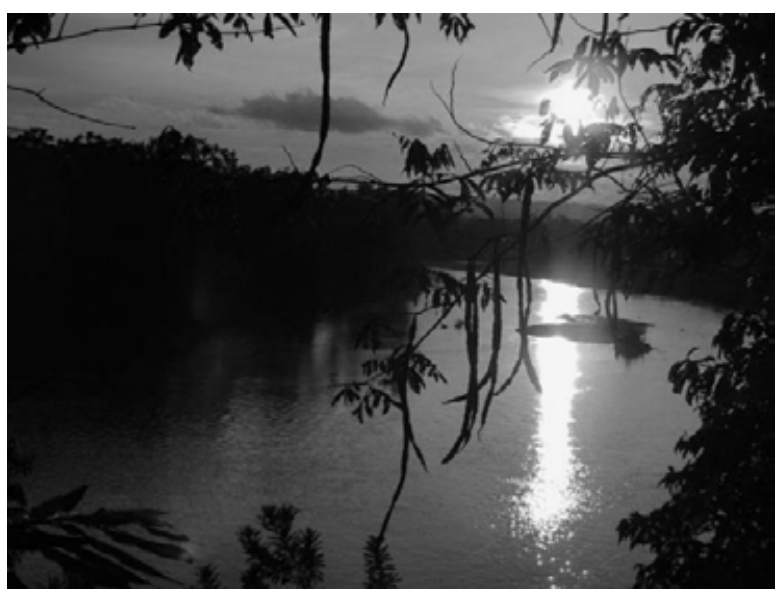

Fotografía 1. A orillas del río Guayuyacu. Baja Bota Caucana.

Fuente: Rodríguez, 2018.
La expresión "saber vivir alli" o ser enseñado a vivir allí, implica formas particulares de relacionarse con nukanchipa alpa mama (territorio y cosmovisión). Para este caso se abordan desde la palabra cosechada con los Inga y desde miradas andinas que lo posicionan como principio de vida y resistencia frente a modelos de gobierno capitalistas, globalizantes y coloniales.

Podríamos caracterizar este principio en atención a que los Inga con sus formas de vida expresan una relación íntima entre lo que nombran y hacen, y que de ello uno de los elementos que da cuenta es la posibilidad de conocer, porque conocer es necesariamente vivir. En palabras de Esterman:

la tarea y capacidad básica del runa [persona-colectividad andina] consisten en el poder de presentación simbólica del cosmos mediante formas rituales y celebrativas. La celebración y el ritual son para el hombre andino un acto gnoseológico y ético de primer rango; celebrar el orden cósmico (en forma simbólica), significa conocerlo y conservarlo (1998, p. 198).

Este acto de conocimiento enlaza tres elementos a saber: sentir: iuiachiuai chaiaspa o quedar: samai, que da apertura al conocer: rigsii, saber: iachai y pensar: iuiai. "La persona puede conocer y saber, alguien sabe porque lo ha vivido, lo ha conocido, lo ha andado; o sabe, porque otro se lo ha contado, se lo hizo saber [y por ende lo aplica a su vida]" (Pueblo Inga - Ministerio de Educación Nacional, 2012, p. 8).

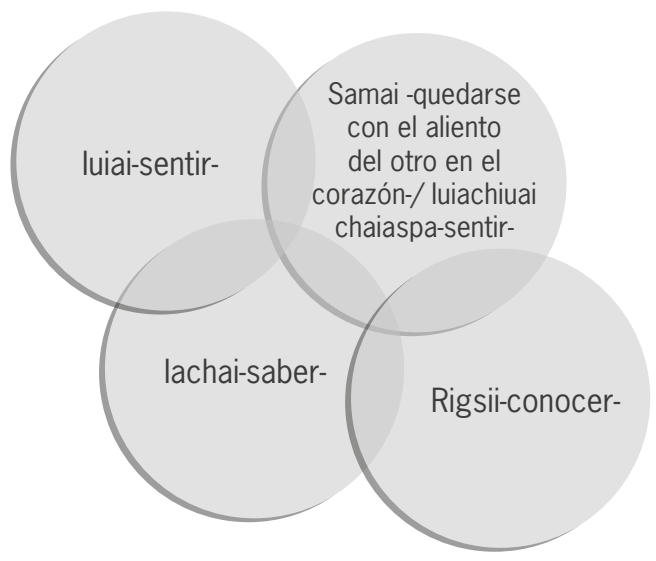

Gráfico 1. Elementos implicados en el acto de conocer, desde el pueblo Inga.

Fuente: elaboración propia. 
Sentir, saber y pensar están posicionando ese anudamiento profundo e inseparable entre vivir $y$ conocer "conocer algo, significa sobre todo: realizarlo celebrativo y simbólicamente (nachvollziehen). En y a través del ritual, se presenta el conocimiento que no es un depósito de la persona, sino una relación intrínseca del plan cósmico" para recrear la vida (Esterman, 1998. p. 168).

Lo anterior implica que todos somos conocimiento que se va desenvolviendo a medida que se vive. Ser persona de conocimiento entonces implica ser chakana:

la función como chakana se manifiesta de muchas maneras: como agricultor, tiende un puente entre los fenómenos de hannaq pacha [mundo de arriba] (lluvia, sol) y la pachamama. Como pareja establece el nexo entre lo femenino y masculino, la izquierda y derecha. Como homo celebrans restituye el orden cósmico violado y desequilibrado. Como ente fecundo establece lazos entre las generaciones y contribuye a la conservación de la vida. (Esterman, 1998, p. 200)

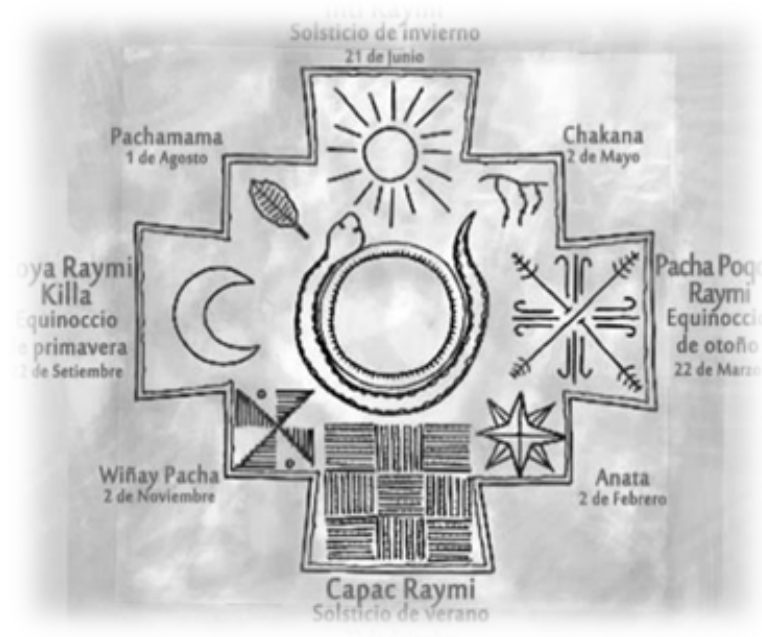

Ilustración 2. Representación de una chakana (cruz del sur) relacionada con momentos astronómicos.

Fuente: https://bit.ly/2Xcp10t

Es así que el consejo de los mayores Inga es "vivir pensando, saber ver [encontrar dentro de uno mismo, cuál es el significado de lo que está viendo], prevenir para actuar o decir, no andar llamando cosas que no se deben estar trayendo" (Bravo, 2010).
Se trata de ir tejiendo con pensamiento, que es la lanzadera, la macana que va pasando por el tejido urdido, por los caminos de conocimiento y, de esta manera, va configurando el tejido de la vida de manera individual y colectiva.

Este tejido de la vida implica unos amarres al origen como garante de ese kutey (eterno retorno al origen) al kosko (ombligo). Se debe aclarar que ese retornar no solo son ciclos o repeticiones de lo mismo, "sino una nueva manera de ordenar el universo" que atiende a las experiencias vividas. Es así que cada runa (persona) tiene sus propios amarres, que son también los conocimientos que trae sembrados y los que se siembran al andar por la vida.

Estos amarres son reflejo de la relación práctica-vida cotidiana; son prácticas en el territorio para la concepción - preparativos de acuerdo a las fases lunares-, la gestación - hablarle en idioma, escuchar cantos y silbidos de los iachagkuna, participar en ceremonias con plantas medicinales...- en el nacimiento - el entierro de la placenta, corte y entierro del ombligo, el corte de cabello- y durante toda la vida - ceremonia de Ambiwaska, sembrar, tejer, celebración del Atun Puncha o Wata Puncha (del vocablo watay -amarrar- ...) - .

Amarrarse al origen, a nukanchipa alpa mama (nuestro territorio), a la tulpa (fogón) es sembrarse en la chagra y en general en el territorio.

El cultivo es entonces una forma de culto, una presentación simbólica del orden orgánico y relacional de la vida. Por eso el trabajo para el campesino andino no es simplemente un acto productivo (o la creación de plusvalía), sino un diálogo íntimo e intensivo con las fuerzas de la vida, una oración a la Pachamama, un acto simbólico de carácter cultico y ritual. (Esterman, 1998, p. 199)

Pero no solo se cultivan alimentos, el conocimiento se debe sembrar, por eso el que no tiene historia, un canto propio, no puede sembrar. En el acto de sembrar, no se puede separar el conocer del saber, solo así se puede transmitir la energía, el espíritu del conocimiento. Una vez sembrado se debe activar, poner a prueba en el hacer, por ello 
en el conocimiento sentir, pensar, hacer, son complemento (Pueblo Inga - Ministerio de Educación Nacional, 2012, p. 8).

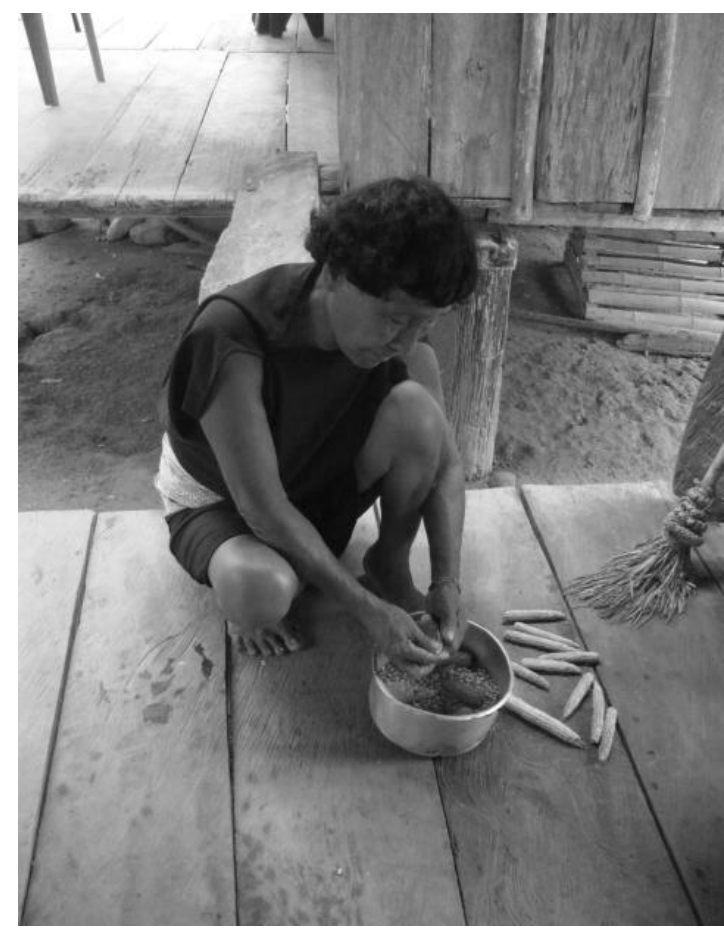

Fotografía 2. Mayora Pastora Jacanamijoy desgranando maíz. Baja Bota Caucana.

Fuente: Bravo, 2018

Esta actividad de siembra también permite cosechar relaciones de reciprocidad y complementariedad con los otros seres y existencias de nukanchipa alpa mama, por ejemplo, el acto de misiar, el intercambio de productos de la cosecha, trabajos colectivos como la minga y el divichidu pueden ser comprendidos como escenarios de relación que al ser orientados por esos principios son "garantía para la continuidad de la vida, en especial, y del orden cósmico, en general" (Esterman, 1998, p. 179).

4 Es decir, entre el canasto donde se llevan las mazorcas se pone, por ejemplo, una de maíces morados. Si la persona no se da cuenta de esto, el vecino le dice misiado, "por lo cual le corresponde al año siguiente, ir donde el misiador, o invitarlo para hacerlo compadre 0 afianzar la reciprocidad con otro producto misio, en medio de una gran fiesta con baile, buena comida y aguardiente" (Mamián, 2004, p. 115).

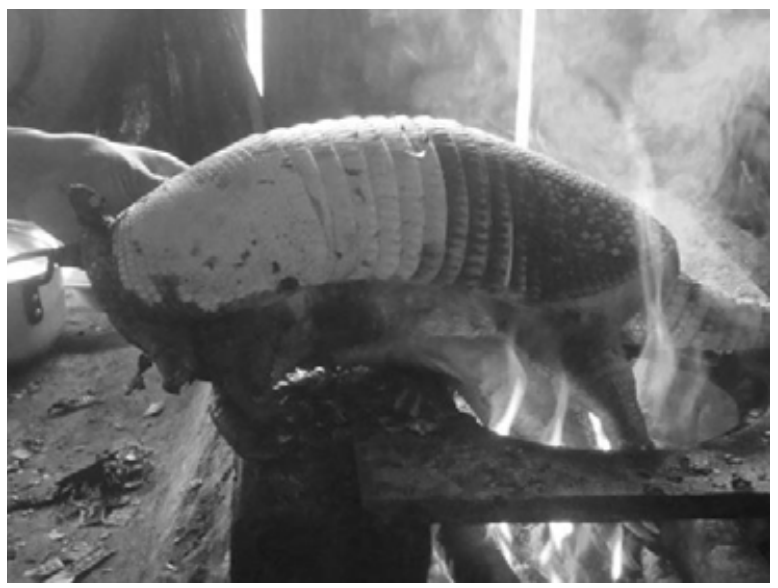

Fotografía 3. Preparando un armadillo para el almuerzo.

Tomada: Bravo, 2013.

Dicho orden se mantiene, por ejemplo, cuando al cazar echan las tripas del animal al río o cuando antes de beber chicha "achican" le ofrecen un chorro al suelo en agradecimiento por la cosecha recibida; también, cuando expresan: "sembrar es sembrarse uno mismo, así como uno las va cuidando [a las plantas] ellas también lo van cuidando a uno". Clara muestra de sus formas de armonización y recreación de la vida.

También en el tejido del chumbe (faja tejida con las de colores, para abrigar el vientre, cuidar la vida) los Inga se atan al origen, a la vez que están atando al hannaq pacha (mundo de arriba) con el kay pacha (mundo horizontal o de aquí), manteniendo el orden cósmico. Es por ello que simbólicamente cuando lo tejen o lo leen siempre está enrollado, ya sea en su cintura o en sus manos, y hay una parte que cae, que tiene contacto con pacha. Este contacto también se hace a través del propio cuerpo del tejedor, quien en ese momento nuevamente es chakana (cruce de mundos). Asimismo, el chumbe puede asociarse también con el kuychi (arcoíris) que hace enrollar pacha o permite kutey; es decir, el mismo concepto expresado en distintas formas.

A propósito ese principio de "saber vivir alli" o estar enseñado a vivir allí nos ubica en debates sobre las formas en que estas comunidades conocen, sienten, piensan y dicen el mundo; no como algo externo a ellos o algo que hay que "dominar, controlar, 
manipular", sino necesariamente como un par con el que se dialoga, se concierta y se recrea la vida. Pensamientos que se distancian profundamente de la ética ecológica contemporánea de Occidente, en donde "hay que cuidar la naturaleza, con el fin de conservar el medio ambiente para la vida humana" (Esterman, 1998, p. 231).

Como expresa Aida Lucía Jacanamijoy:

el vivir no solo es un verbo, sino que tiene trascendencia de vida como tal. Lo que conlleva a decir "vivo" es que todo lo que está alrededor tiene más que vida o sea la humanización de mi territorio, como interactúo y me complemento.

Para reiterar que el lugar del runa (persona) como chakana importante del cosmos, se caracteriza por su

función cósmica de conservación y perpetuación ritual y ceremonial [de la vida]. Por lo tanto, no es un fin (telos) en sí mismo, sino una cofinalidad cósmica. El ser humano es parte intrínseca del cosmos (pacha); esto justamente le da su dignidad y posición excepcional (Esterman, 1998, p. 231).

A propósito de esas complementariedades, podríamos mencionar cuando los Inga plantean esa relación entre los seres y existencias medicinales del Alto Putumayo - (páramo, montaña) medicinas frías para enfermedades que tienen que ver con aumento de calor en el cuerpo- con los del Bajo Putumayo, Caquetá y Baja Bota Caucana (ввс) -medicinas cálidas para enfermedades que tienen que ver con aumento de frío en el cuerpo- - También, cuando mencionan que "desde antigua los [Inga] del Alto traían sus chumbes para intercambiarlos por productos del Bajo [Putumayo]", esta función de la persona andina expresa su comprensión de la vida como ese "flujo de energía cósmica en y a través de oposiciones complementarias" (Esterman, 1998, p. 231).

En los relatos de mayores, sabedoras, sabedores $y$, en general, de la comunidad Inga, siempre está presente la concepción de que todos los seres y existencias de Pacha son vivos y se complementan entre sí, y en esa medida son expresión de relaciones armónicas.
Recordemos el consejo a la madrugada durante uno de los recorridos por ввс:

que no se nos olvide que las piedras son gente como nosotros. Que ellas están ahí cuidando del territorio. Que cómo se nos ocurre echarles barro en sus ojos, en sus cuerpos, ensuciando sus casas, además que ¿quién dijo que las algas que las recubrían eran mugre, como para quitárselas? [...] la bifurcación occidental entre lo vivo y no-vivo, lo orgánico e inorgánico, lo animado e inanimado, lo humano y no-humano, no es una concepción transculturable al ámbito andino (Esterman, 1998, p. 176).

Nukanchipa alpa mama también se comprende como otro ser y sus dinámicas de organización y existencia se asemejan con el propio cuerpo. Así, cuando mencionan "La unión del camino lo relaciono con nuestro cuerpo, así mismo el territorio [...] si nos cortamos una vena, lo afectamos todo, así pasa con nuestros caminos" (Bravo, 2010) nuevamente nos invitan a relacionar la vida y lo vivo con todo aquello de lo cual somos parte. Como menciona Reynaga (2007) "Nadie es indispensable y todos son necesarios" para mantener la armonía cósmica, para vivir bien:

Es recuperar nuestra vida en completa armonía y respeto mutuo con la madre naturaleza, con la Pachamama, donde todo es vida, donde todos somos uywas, criados de la naturaleza y el cosmos, donde todos somos parte de la naturaleza y no hay nada separado, donde el viento, las estrellas, las plantas, la piedra, el rocío, los cerros, las aves, el puma, son nuestros hermanos, donde la tierra es la vida misma (Choquehuanca, 2010, p. 10).

Recuerdo una conversación con Aida a principio del año 2015, en la que me explicaba cómo hablar de saber vivir ahí puede estar relacionado con el suma kaugsay (vivir bien), porque realmente a lo que está haciendo referencia es a un principio de vida complementario con todos los seres y existencias del territorio.

Este comentario tramado con los planteamientos realizados en esta investigación posiciona el abordaje de este principio de vida Inga con debates 
académicos relacionados con "Vivir bien o un buen vivir o Sumak Kawsay, Suma Qamaña, Balu Wala, en las lenguas de algunas culturas originarias de Abya Yala (la tierra grande donde todos vivimos)" (Ibañez y Aguirre, 2013), en donde ubican este principio de vida como alternativa a la crisis de paradigma que estamos viviendo en estos momentos.

Dicha alternativa denominada por el historiador aymara, Fernando Huanacuni, en Dávalos "el paradigma de la cultura de la vida", se propone como opción ante los dos paradigmas propuestos por "occidente":

a. Uno individual extremo (individualismo): el paradigma individual, es el que está vigente y determina las relaciones sociales, jurídicas y de vida actual; desde hace siglos este paradigma está llevando a sociedades de todo el mundo hacia una desintegración, debido a un alto grado de desensibilización de los seres humanos. Esto ha tenido consecuencias a todos los niveles, ha ido depredando la vida en su conjunto. Para este paradigma, lo más importante es la acumulación del capital.

b. Uno colectivo extremo (comunismo): para el paradigma colectivo extremo, como el comunismo, el socialismo, el bienestar del ser humano es lo más importante, sin tomar en cuenta las otras formas de existencia (2008, p. 17).

El paradigma de la cultura de la vida, caracterizado por la visión de que

todo está unido e integrado, y que existe una interdependencia entre todo y entre todos (...) es la posibilidad de vincular al hombre con la naturaleza desde una posición de respeto; porque es la oportunidad de devolverle la ética a la convivencia humana; porque es necesario un nuevo contrato social en que puedan convivir la unidad en la diversidad; y porque es la oportunidad de oponerse a la violencia del sistema (Dávalos, 2008).

Comprende una crítica a los planteamientos actuales sobre el "desarrollo armónico, desarrollo con identidad, los ideales de vivir mejor en vez del
Vivir Bien", en vez de buscar alternativas al desarrollo. "las diferencias son claras: el vivir mejor significa vivir a costa del otro, explotando al otro, saqueando los recursos naturales, violando a la Madre Tierra, privatizando los servicios básicos; en cambio el Vivir bien es vivir en solidaridad, en igualdad, en armonía, en complementariedad, en reciprocidad" (Ibañez y Aguirre, 2013, p. 65).

Esto no significa que desde esta perspectiva no existan conflictos y disensos, lo que se propone son otras formas de entender las relaciones entre todos los que hacemos parte del cosmos y de resolver las tensiones que de allí surgen. Es la posibilidad de construcción colectiva de otros órdenes y equilibrios que permitan la vida; cuestiones que, para el caso de Bolivia y Ecuador, se han integrado en parte de sus dinámicas y debates constitucionales, de país, académicos y que en el caso colombiano apenas asoma en el decir de algunos.

\section{Saber vivir en la baja bota caucana}

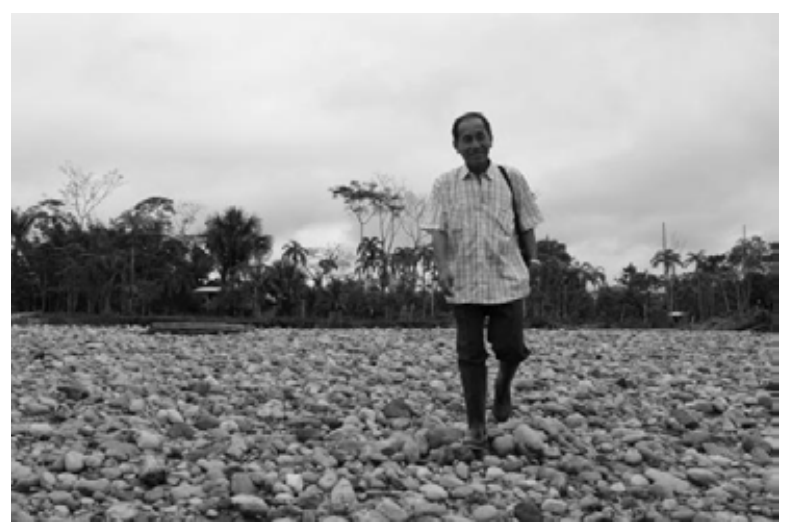

Fotografía 4. El mayor Eusbio Peña, recorriendo su territorio. Baja Bota Caucana.

Fuente: Bravo, 2013

Dice don Eusebio: "hay que tener calma, todo pasa”. Y es que sobreponerse a la complejidad de sus situaciones de existencia en estos territorios implica la presencia de un pensamiento de transformación "que tal vez nuestros ojos no alcancen a ver, pero que se anuncia en cada ritual, en cada canto, en cada saludo en nukanchipa rimai (nuestro idioma), en cada encuentro con nukanchi runa (nuestra gente)". 
Esta afirmación implica una manera de posicionarse frente al mundo. Para la sociedad hegemónica aquello de que "hay que esperar que las cosas cambien" se podría interpretar como una actitud de parcialidad y pasividad frente a hechos que afectan la existencia; pero al contrario de esto, lo que se espera por parte de la comunidad Inga, representada para el caso de este trabajo en la palabra de los mayores, es un eterno retorno al origen (kutey), donde el tiempo es cíclico, en lugar de lineal:

ciclos que no son meras repeticiones o retornos de lo mismo, sino una nueva manera de ordenar el universo (pacha), bajo ciertos parámetros. [...] La diferencia más importante con el pensamiento dialéctico de occidente radica en el rechazo de la progresividad inherente al proceso temporal histórico. [...] [En el pensamiento andino] el pasado (ñawpa pacha o wiñay) está presente en el tiempo actual (kay pacha o wiñay) de distintas maneras. Los antepasados no han dejado simplemente de existir o influenciar, sino que siguen 'viviendo' dentro del pueblo (Esterman, 1998, p. 185).

A partir de esta mirada podríamos identificar algunos elementos de análisis que nos permiten acercarnos a la comprensión de la temporalidad del Inga en este caso, elemento que reafirma la hipótesis de que los Inga han establecido formas particulares de "estar allí", de "vivir allí". Estos elementos son:

a. Los Inga no son superiores a la naturaleza, hacen parte de ella.

b. Los Inga están esperando el momento propicio para el cambio (pachakutik).

c. Ellos entienden unos tiempos de la naturaleza, que no son los tiempos impuestos por las lógicas del mundo contemporáneo, que se encuentran mediados por los procesos de producción de la mercancía y la reproducción del capital.

d. Para los indígenas no existe un progreso hacia el futuro, no se inscriben en las lógicas contemporáneas del "desarrollo".

e. Esta mirada implica entender que el territorio desde el origen ya está ordenado.
Ahora bien, esperar no significa no hacer nada. Al contrario, esperar se puede comprender como una forma particular de hacer, de tejer posibilidades de existencia en el territorio desde las prácticas cotidianas, sin que ello implique transformaciones radicales de sus modos de existencia. Hay múltiples formas de reaccionar frente a los problemas de la vida, una de ellas es esperar, pero no de forma estoica, sino activamente desde la concreción de una práctica de la vida cotidiana que reafirma una manera particular de "vivir alli".

Por ejemplo, para los Inga hay posibilidades que se activan cada vez que, abrigados por la tulpa (fogón), se reúnen en familia para comer rayana - sopa a base de plátano rayado (en rayador de palo), con trozos de yuca, cilantro cimarrón, cebolla larga y proteína (pescado o carne de monte)_; maito cusado - pescado suasado en hoja de sirindango (palma), condimentado con cilantro de monte, cebolla larga y pepa de sirindango-; uchú manga - caldo de ají, preparado con las tripas de la gallina, o del pescado-, caldo de pescado, envueltos o un plátano asado. Compartiendo chicha de maíz, anduche (chicha de yuca), putu Alcides (fermentado de plátano) o una chucula - bebida a base de chiro (plátano) maduro-

Lo que aquí se expresa es que para los Inga el acontecimiento de "comer estos alimentos" es producto de una serie de relaciones y actividades que sostienen con el territorio y con su cultura. La importancia de sembrar sus alimentos, entonces, se puede interpretar desde los siguientes elementos:

a. Como dice Esterman (1998), el hombre Inga es co-creador de la casa común de todos. El hombre es ante todo "agri-cultor" y no "productor", es decir, cuidador de la casa (wasikama).

b. Comer y beber "comida buena" es hacer culto a la tierra.

c. No solo se alimenta el cuerpo físico, sino que se alimentan las relaciones sociales a partir del cuidado del cultivo, de la chagra.

Lo que se evidencia es que en el acontecimiento de "comer esto" volvemos a encontrarnos con la expresión de esos tres elementos descritos como 
constituyentes de la filosofía andina: relacionalidad, reciprocidad y complementariedad, los cuales se explican a continuación, en clave de las relaciones que el Inga establece con la chagra, la cosecha y la comida, elementos propios de su cotidianidad a partir de los cuales podemos seguir desarrollando lo que implica "saber vivir ahí" para los Inga:

a. Relacionalidad, debido a que el acto de sembrar, de trabajar la tierra implica ser parte de nukanchipa alpa mama y, en esa medida, únicamente se puede ser en relación con los otros seres y existencias. Por eso cultivar es manifestación de esas relaciones no solo con el suelo como sustrato de las plantas, sino también con elementos como el oxígeno, el agua, el sol, la luna y los otros Inga de la comunidad; pues, como se viene planteando, sembrar implica también coordinar trabajos con otros integrantes de su pueblo que pueden ser la esposa, los hijos, los parientes cercanos, pero también otros que pueden ser personas de la comunidad invitadas a los trabajos colectivos, como la minga.

b. Reciprocidad, debido a que al cuidar a las plantas ellas también los cuidan a ellos, no solo proporcionándoles alimentos, sino curando enfermedades y protegiéndolos espiritualmente. Esta práctica se ve reflejada en el territorio con la participación de los Inga en actividades como la toma de yoco, verbena y Ambiwaska, prácticas de medicina tradicional que posibilitan mantener el cuerpo físico y la espiritualidad en armonía con el territorio. Estas prácticas están acompañadas de los "consejos a la madrugada", que son palabras que orientan a la persona para la toma de decisiones individuales y colectivas y que generalmente los dan los mayores y mayoras de la comunidad.

Otro ejemplo de estas relaciones de reciprocidad y complementariedad son los esfuerzos de los mayores Inga por mantener sus chagras con medicinas tradicionales, por resguardar así sea una "montañita" como la que cuida don Eusebio Becerra (Mayor del resguardo Inga San Rafael), bajo el entendimiento de que al sembrar plantas y cuidar de ellas también se está cuidando la propia vida y la de los Inga de la comunidad, pues allí están sembrando además de alimentos, medicinas para curar a sus enfermos. Es una comprensión, además, de que, al cuidar la capa vegetal de la montaña, se están cuidando los nacederos de agua, se están cuidando los ríos y los peces que hay en él. Este cuidado se ve reflejado, por ejemplo, en buena carne para la cacería y aguas limpias para beber y bañarse.

En síntesis, en el marco de la reciprocidad y relacionalidad, si se siembra una planta y se deja marchitar, asimismo esto se verá reflejado en la falta de cosecha, por ende, en la falta de alimentos para quien sembró y su familia. Además, al no tener cosecha para compartir, intercambiar u ofrecer, también se están afectando las relaciones sociales con los otros integrantes de la comunidad, así como su espiritualidad.

c. Complementariedad, pues al conocer las dinámicas de crecimiento y desarrollo de las plantas, los Inga también han identificado qué tipo de plantas deben estar al lado de otras para; por ejemplo, brindar protección ante un insecto hospedero, qué plantas le ayudan a fijar mejor los nutrientes del suelo, cuáles ayudan a mantener reservas de agua. De manera que indica no solo relacionalidad entre los diferentes elementos del territorio, sino complementariedad entre las plantas.

Otro ejemplo en este sentido son los intercambios de plantas medicinales de tierra fría que realizan los Inga del Alto Putumayo con los del Bajo Putumayo, Baja Bota Caucana y Caquetá, quienes les ofrecen, en cambio, de esas plantas, medicinas encontradas en el piedemonte amazónico.

Otro elemento que aporta para la comprensión de esa idea de que "esperar a que los tiempos cambien" no es quedarse sin hacer nada son las actividades de cuidado de la fauna, elemento constitutivo de lo que los Inga llaman "seres y existencias" del territorio. Don Eusebio señala frente a este aspecto 
que tiene al otro lado del río Fragua un criadero de yulo, boruga y gurre (armadillo), los cuales son animales que han sido dejados en esa parte del resguardo para cuidarlos y que se reproduzcan, para luego tener carne de cacería y poderse alimentar con sus recetas tradicionales.

En este proceso interviene no solo él como cuidador de dichos animales, sino toda la comunidad del resguardo, quienes tienen el compromiso de no cazarlos hasta que se hayan reproducido y el territorio este repoblado. Como lo vimos anteriormente en uno de los relatos de don Eusebio, para conseguir que estos animales salgan de la casa del sol, donde están todos los animales "guardados" (Indi wasi) hay que hacer una concertación con los animales a través de los iachagkuna (sabedores de la medicina ancestral Inga) y todo depende del cumplimiento de los compromisos pactados - correspondencia en las acciones - y de la posibilidad de concertación con los campesinos y colonos, "que atrevidamente ingresan al territorio del resguardo con el objetivo de cazar con escopeta, acciones ante las cuales deben estar alerta", dice don Eusebio.

Hay que señalar que estas acciones "atrevidas" de las que habla don Eusebio relacionadas con la caza de animales y la tala de especies arbóreas que están en proceso de repoblamiento en los territorios de los resguardos no solo se dan por parte de campesinos y colonos, sino también por parte de integrantes de las mismas comunidades indígenas que no respetan los acuerdos pactados para el cuidado de las especies que históricamente se han integrado a prácticas culturales como la caza, contribuyendo a la desarmonización de nukanchipa alpa mama (territorio).

Es oportuno mencionar que aunque en Colombia existe una política que reglamenta el uso de los recursos de estos territorios y de su diversidad biológica, desde la práctica cultural los Inga han establecido unos modos propios de relacionamiento y de regulación del territorio; modos que se expresan en situaciones como las señaladas por don Eusebio, quien declara que "al interior de la comunidad se establecen pactos para el repoblamiento de los territorios" de especies que, por efectos de la caza y la tala indiscriminada (por parte de colonos, campesinos e Ingas), vienen disminuyendo sustancialmente en población, poniendo en peligro la diversidad biológica, cultural y social de estos territorios.

Cuando no se cumplen los pactos, los iachagkuna (sabedores de la medicina ancestral Inga) no pueden "seguir concertando con los espíritus de los animales y las plantas" para que vuelvan al territorio, y por ello en estos momentos es poco probable ver este tipo de organismos andando en el territorio. Estas son cuestiones que, por supuesto, no son contempladas en las legislaciones ambientales.

En este sentido hay que señalar la distancia que existe entre los planteamientos de la normatividad y las realidades cotidianas de comunidades en territorios específicos, para el caso nuestro, los Inga. Con ello se genera una respuesta activa de las comunidades mismas, quienes en consenso entran a definir esos otros tipos de regulaciones que requieren las comunidades para su permanencia en el territorio. Por ejemplo, comenta don Eusebio, "no contándole a la gente que los animales están por ahí, evitando que la gente se entre a los territorios a cazar", acción que en ocasiones genera tensiones entre los Inga con campesinos y colonos, y entre los mismos Inga.

\section{Saber vivir como práctica cambiante}

Saber vivir allí es una práctica de vida de los Inga y, como tal, cambia en el devenir histórico. Interactúa con los actores sociales e intereses presentes en el territorio: colonos, campesinos, funcionarios del Estado, investigadores y comunidad en general. También, con la diversidad de realidades que se encuentran en sus traslados y desplazamientos por el territorio nacional (Bogotá, Mocoa, Caquetá, Pasto, Villavicencio, Valledupar, Medellín) e internacional (Venezuela, Ecuador, Bolivia y Estados Unidos, principalmente).

Un ejemplo de estas dinámicas del principio "saber vivir allí", es la apropiación de un discurso evidentemente estatal, como lo es "la seguridad alimentaria”. El cual se integra en el marco del diseño de sus planes de vida y que para el caso de los Inga de la ввс inicia con un diagnóstico donde definieron: 
cuáles son los espacios de uso del territorio para procurarnos alimentos -las chagras, la montaña, los rastrojos, las quebradas y ríos, entre otros-. También definimos cómo habían sido los sistemas productivos hace más de 50 años y cómo son ahora. Reflexionamos sobre los recursos naturales y el estado de conservación de los territorios. [...] Identificamos las problemáticas, las necesidades y las alternativas de solución (Pueblo Inga de la BвC, 2013, p. 18).

Ejemplo de lo anterior pueden ser los programas de sustitución de cultivos ilícitos, puesto que estos apuntan a la siembra de monocultivos como: palma de aceite para generar biocombustible, árboles maderables y plátano. Estas propuestas han ocasionado que los Inga sigan incursionando en sistemas productivos que dependen de los agroquímicos, semillas mejoradas o modificadas genéticamente, dependencia del mercado para la venta de sus productos, situaciones que impactan sus formas culturales de relacionamiento en el territorio y van en detrimento del mismo.

Esto, además de afectar la diversidad biológica de los ecosistemas del piedemonte amazónico, reflejado en pérdida de semillas nativas, acidificación del suelo y resecación, afecta las relaciones culturales y sociales en sus comunidades y, por esa vía, atenta contra su posibilidad de seguir viviendo en esos territorios.

Estas situaciones ponen en evidencia la necesidad de que ese principio de "saber vivir allî" se reconfigure de cara a las nuevas situaciones que se están presentando en la cotidianidad de sus vidas. "Saber vivir allî" también significa tener la capacidad de validar el contexto, interpretarlo y actuar en consecuencia con él, sin que ello implique dejar de ser Inga.

Ahora bien, como el principio de saber vivir allí no es estático, su desarrollo también implica una serie de tensiones $y$, si se quiere, de contradicciones, que se ven reflejadas, por ejemplo, en prácticas económicas que la comunidad Inga ha querido incorporar a sus formas de vivir. Es el caso de la formulación e implementación del Plan Sectorial de Desarrollo Agropecuario Municipal (Periodo 2012-2015) (en el que participan representantes de los cabildos Inga de la BBC), que está impulsando el monocultivo de plátano AA (píldoro ó bocadillo) en miras a su comercialización.

Cuestión que entra en detrimento de la economía de los pobladores de la región, pues no es garantizado el proceso de comercialización; es decir, aunque se obtengan grandes cosechas de plátano, no hay un plan claro de transporte y venta del producto, arriesgándose a que nadie les compre sus cosechas o, si lo hacen, sea a menor costo de lo que se invierte para obtenerlas.

En un intento por superar esas contradicciones y tensiones, la Asociación Atunkunapa Alpa (La tierra de nuestros mayores) ha generado estrategias como la de apoyar la formación en temas de agroecología, con el ánimo de que se formen en las estrategias de siembra y manejo de cultivos más apropiadas para cuidar la diversidad biológica del territorio; esta incluye la conversación con los mayores y mayoras Inga de las comunidades para recordar las formas ancestrales de cultivar, recuperar semillas nativas y la formación desde perspectivas de las agriculturas sostenibles, en aras de poder activar conocimientos contextualizados a estas épocas y circunstancias.

Esa activación de conocimientos implica no solo que estas personas se formen, sino que además salgan de correría por cada una de las comunidades. Caminar por el territorio se hace en permanente conversación con abuelos y abuelas, respecto a las formas ancestrales de siembra, cuidado de los cultivos y en general cuidado de la vida en el territorio.

De esa manera se incentiva la renovación de memoria respecto a sus conocimientos sobre el cuidado de nukanchipa alpa mama, la construcción de argumentos para la toma de decisiones frente a las políticas de desarrollo del municipio y, por esa vía, aportar al fortalecimiento cultural a través de cultivar "comida y bebida buena"; lo cual implica en el marco del principio "saber vivir alli":

a. Fortalecer la siembra de sus chagras, en la medida en que a este espacio se le entiende como escenario de recreación de la diversidad; reflejo de esto la variedad de productos sembrados en ella: yuca, ñame, bore, piña, plátano, borojó, caña, maíz, plantas aromáticas 
y otras de carácter medicinal, como la verbena, limonaria, boldo, diente de león, etc., y algunas variedades de plantas rituales, como el yoco.

b. Reducir el uso de agroquímicos gracias a sus conocimientos sobre las dinámicas de relación entre las plantas (complementariedad y relacionalidad).

c. Los cuidados y manejos espirituales para que las semillas den fruto.

d. Las relaciones sociales que allí se fortalecen como el trabajo conjunto, compartir la cosecha y preparación de platos tradicionales.

e. El fortalecimiento de sus relaciones comunitarias, a través de la implementación de los proyectos de recuperación de semillas nativas $\mathrm{y}$ formas ancestrales de cultivar.

En síntesis, los elementos que en este apartado se exponen con relación a lo dinámico del principio "saber vivir alli", de cara a su encuentro con la sociedad hegemónica, tienen que ver con los siguientes elementos:

a. Generar debates al interior de las comunidades y cabildos, respecto a los impactos $\mathrm{y}$ transformaciones que puede generar el encuentro con otras culturas y sociedades.

b. Resignificación del ser Inga, que aporta a los procesos de reconfiguración del "saber vivir alli'".

c. La apropiación estratégica de discursos institucionales que les posibilitan entablar diálogos con la cultura hegemónica y tramitar recursos para adelantar proyectos en los territorios. Esto no impide que se desarrollen sus formas propias de abordar la importancia cultural de la siembra, cosecha y consumo de alimentos tradicionales. Ese tipo de discursos es otra de las expresiones de ese mestizaje, en donde se cruzan múltiples sentidos culturales.

d. Encuentro de las prácticas socio-culturales de los Inga con otras formas de estar en el territorio, para la activación de conocimientos contextualizados a sus realidades de vida. e. Posibilidad de ingreso de su cosmovisión en el debate público local y nacional de su mirada particular, sobre lo que implica saber vivir en un territorio específico.

\section{Conclusiones}

- El principio "saber vivir allî" es una noción que se hace reiterativa en la cotidianidad de los mayores y mayoras Inga. Sumado a esto, el hecho de entender a los mayores y mayoras como personas de conocimiento que han vivido unas experiencias de vida que les permiten orientar la toma de decisiones para la vida en comunidad hace que, para el caso de este trabajo, sus palabras sean las que estructuran la caracterización de este principio.

- Teniendo en cuenta que el principio "saber vivir allî” de los Inga es dinámico, que se reconfigura de acuerdo a unos contextos, actores e intereses específicos, puede ser entendido como una apropiación y transformación del discurso sobre territorio. En este sentido "saber vivir alli" se relaciona con elementos constitutivos de la categoría territorio como:

- aspectos de ordenamiento territorial: mapeos, limitación de territorios, saneamiento territorial, constitución en cabildos y resguardos;

- formas de construcción de vivienda, cacería y pesca, preparación de alimentos, manejo y uso del suelo, formas y tipos de cultivo, actividades espirituales;

- impactos sobre el territorio, en palabras de los Inga "desarmonización de nukanchipa alpa mama", debido a la presencia de familias cocaleras, presión a la oferta ambiental, incursión en economías ilegales, participación en proyectos que fomentan el monocultivo;

- y organización social (formas de gobierno propio, medicina tradicional).

El principio "saber vivir alli" no solo retoma elementos de la categoría territorio, sino que, además, deja ver las tensiones que surgen con el uso de esta categoría, en tanto que: 
- No está claro por qué desde las políticas nacionales para territorios indígenas se contempla la necesidad de ordenar el territorio, pues para ellos el territorio desde el origen ya está ordenado. En este momento lo que alcanzaría a ser es un reordenamiento del territorio.

- Ese reordenamiento del territorio implica unos pactos con los dueños de los animales y demás seres y existencias del territorio, acciones que desde la legislación política y ambiental no se contemplan.

- Las ideas de "progreso y desarrollo" que se imponen desde las políticas económicas y de gobierno que rigen nuestro país excluyen los conocimientos que sobre el territorio tienen las comunidades, esto agudiza los problemas $y$, sobre todo, su falta de éxito al ser implementadas en los territorios.

- "Saber vivir alli" como principio de vida para los Inga se plantea como opción ante las ideas de "progreso y desarrollo" impuestas por el colonialismo y neoliberalismo. Esta opción de vida, está caracterizada por:

- La posibilidad de entender que las personas son parte del universo, de la naturaleza; son wasikamakuna (cuidadores del territorio) y no sus dueños y explotadores.

- No hacer oposición entre los "seres vivos" y los "no vivos", lo que permite otorgarle a la naturaleza y en general a nukanchipa alpa mama (territorio), el estatus de ser vivo, quien merece respeto, cuidados, además de ser digna de conversar con ella, para llegar a acuerdos sobre su manejo y protección.

- No polariza ni contrapone, las categorías bueno y malo, las asume como unidad complementaria; es decir, no puede haber lo uno sin lo otro. Esto se refleja en la posibilidad de participación que tienen todos los seres y existencias en la toma de decisiones sobre el territorio, como lugar de la vida.

- Una mirada sistémica del territorio, lo cual permite no segmentar la realidad ni dotar de mayor estatus a los humanos dentro de la red de relaciones que sustentan la vida.

- Posicionar los referentes contextuales locales frente a los intereses de homogenizar las realidades, proponiendo por esta vía que las intervenciones en los territorios deben ser particulares y atiendan a la diversidad social, cultural y biológica que subyace a cada territorio.

- Con esto también está posicionando la importancia de esos otros lugares, de esas otras formas de vivir, pensar, conocer y relacionarse con el mundo, para la construcción de Estados, países y políticas que tengan en cuenta la diversidad no como problema sino como fortaleza para la construcción de otros sentidos.

- Entender que si todos somos parte del todo a cada acción le corresponde otra, lo que implica asumir que se comparte la responsabilidad de cuidar la vida. Todos estamos en la posibilidad de armonizar las situaciones que ponen en desequilibrio la vida a través del trabajo, de la relación con la tierra, con el cultivo, con los demás seres y existencias. Esto se convierte en posibilidad de conocer y por ende de mantener la vida.

- Entender que todos somos conocimiento desplaza algunas relaciones de poder jerárquicas en lo que tiene que ver con quién es el dueño del conocimiento, en qué condiciones se pone a circular y con qué intenciones.

- Los encuentros con el otro y lo otro enfrentan a los integrantes de las comunidades Inga a la necesidad de entenderlo y cuestionarlo, ampliando la mirada frente al referente de vida de lo que denominan "sus mayores", ratifican la pertinencia en estos tiempos de conocer e incluso aplicar otras formas de relacionarse con el territorio.

La ruta metodológica construida en esta investigación permite:

- El reconocimiento y valoración de la diversidad de formas de construcción de conocimiento; lo cual implica, para el caso de la 
investigación social, una apertura al diálogo con otros referentes epistémicos y ontológicos.

- La deconstrucción de las relaciones conocimiento/poder tan necesaria en la educación, así como el reconocimiento de la diversidad cultural y biológica del país.

\section{Fuentes orales}

En la Baja Bota Caucana:

Las orientaciones preventivas y visionarias de los caminos del Ambiwaska, con Taita Paulino Mojomboy y su familia: sus hijas Doña Olga y Rubiela Mojomboy, sus hijos Taita Oscar y Alfredo, sus nietos Freider y Nury, sus bisnietos: Carol y el ahijado Andrés Felipe. Resguardo La Floresta- Española.

Don Eusebio Becerra Yapo, mayor del Resguardo Inga de San Rafael (ввс). Transitando los caminos con sus hijos Chucho, María, Juan Pablo y Carolina. Con el yerno Gerbacio (esposo de Carolina) y sus hijos: Dennys, Elkin, Wilson, Brayan y la “Nana”. Resguardo Inga San Rafael.

Profesor Tiberio Muchavisoy, de la comunidad Rumiñawi.

En el Putumayo:

La mama Margarita Jacanamijoy y el taita Francisco "Pacho" Tisoy, médicos Inga del Alto Putumayo, vereda Vichoy.

Aida Lucía Jacanamijoy Muyuy, sus hijos Ipi Kindi y Raimi. Sus padres, taita Justo Jacanamejoy y la abuela Juana Muyuy, quien ya se descansó y desde el otro lugar nos acompaña. Ingakuna de Santiago, Alto Putumayo.

Mercedes Jacanamijoy y su esposo Jesús Carlosama. Ingakuna de Santiago, Alto Putumayo.

Lizbeth Bastidas Jacanamejoy. Inga de Santiago, Alto Putumayo.

Luís López. Inga de Mocoa, Putumayo.

Profesor Alberto Tisoy, de la comunidad Inga Wasipanga, Bajo Putumayo.

\section{Referencias}

Bravo, L. (2010). Diario de campo.

Ciro, E. y Ciro, A. (2008). Del caucho a la ganadería: Transformación del paisaje en el piedemonte caqueteño, 1887-1965. En A. Buitrago y E. Jiménez (eds.). Gente, tierra y agua en la amazonía (pp. 105-132). Universidad Nacional de Colombia- Sede AmazoníaInstituto Amazónico de Investigaciones (IMANI).

Choquehuanca, D. (2010). Hacía la reconstrucción del vivir bien. América Latina en Movimiento, (452).

Dávalos, P. (2008). El Sumak kauwsay ("buen vivir") y las censuras del desarrollo. Cemos.

Delgado, O. y Montañez, G. (2007). Espacio, territorio y región: conceptos básicos para un proyecto nacional. Cuadernos de Geografía, 7(1-2).

Esterman, J. (1998). Filosofía Andina: estudio intercultural de la sabiduría autóctona andina. Abya-Yala.

Ibañez, A. y Aguirre, N. (2013). Buen vivir, vivir bien: Una utopía en proceso de construcción. Ediciones desde abajo.

Instituto Caro y Cuervo. (2000). Lenguas indígenas de Colombia: una visión descriptiva. Instituto Caro y Cuervo.

Mamián, D. (2004). Los Pastos en la danza del espacio, el tiempo y el poder. Ediciones Unariño.

Pueblo Inga de la Baja Bota Caucana. (2013). Ingakunapa Llagta Kaugsai Nambi -Plan de vida-. Piamonte-Cauca.

Pueblo Inga. (2012). Plan de salvaguarda del Pueblo Inga de Colombia: Nukanchipa Kaugsaikunata Iuiaikunata Mana Uañungapa Sakisunchi. Ministerio del Interior.

Pueblo Inga - Ministerio de Educación Nacional . (2012). Kasami Purinchi Nukanchipa Iachaikunawa -Así caminamos con nuestros saberes-. Mocoa: Sin Publicar .

Reynaga, R. (2007). Tawa Inti Suyu: 5 siglos de guerra india -Chukiagu-Marka Koyasullu. Perú.

Vasco, L. (2000). Entre selva y páramo. Viviendo y pensando la lucha india. Instituto Colombiano de Antropología e Historia. http://www.luguiva.net/ libros/detalle.aspx?id=3 